\section{The antenatal diagnosis of fetal anomaly: where to deliver the baby?}

\author{
Martin P Ward Platt
}

The question as to where to deliver the baby arises whenever there is an antenatal diagnosis of significant fetal anomaly. The decision should in theory be made collaboratively between parents, fetal medicine specialists, neonatologists and relevant paediatric specialists (eg, cardiologists, surgeons, nephrologists); but the discussion, and the decision, can be distorted by a variety of factors. These include misperceptions of safety, based on anecdotes of unfortunate experiences; the desire of regional fetal medicine specialists to see the outcomes of their diagnoses; and real or imaginary doubts about the ability of a referring centre to undertake initial stabilisation of a neonate with a potentially complicated problem.

Patient safety is clearly a very important issue, but statements about safety should be based on hard data rather than anecdote or uninformed opinion. For this reason, the paper by Kelsall et al in this edition is a welcome addition to the literature on the safety of delivering babies with antenatally diagnosed cardiac anomalies at a site that, though remote from the cardiac surgical facility, nevertheless has high-quality diagnostic capability and a large neonatal intensive care unit. Essentially, they demonstrate that this practice is safe, though with a number of appropriate caveats - and readers might wonder what happens when their one neonatologist with advanced echocardiography skills is on holiday. These data will become even more important in the UK when the number of cardiac surgery facilities is further reduced, but they will be relevant in many countries outside the UK as well, particularly those where the population is more widely scattered.

We should be wary of allowing safety to dominate the discussion. When patient safety does not clearly require delivery away from the hospital where the mother preferred to deliver before the fetal diagnosis, what other considerations should be taken into account? Parental preferences

Correspondence to Martin P Ward Platt, Regional Maternity Survey Office, 1-2 Claremont Terrace, Newcastle upon Tyne NE2 4AE, UK:

m.p.ward-platt@ncl.ac.uk are clearly important, but those preferences will be influenced by other kinds of information that parents need to hear from the relevant professionals.

Though delivery in the mother's local facility might look superficially attractive, problems arise when the baby is transferred to the specialist centre if the mother, often for obstetric reasons, is not able to travel there straight away; and travel may be further delayed if the referral centre does not have a maternity facility on site (it might be a children's hospital), as the mother will have to be fit for discharge in order to travel there. If the mother has a partner or husband with legal parental responsibility, issues such as giving informed consent for urgent procedures can be managed relatively easily as this person will usually come to the referral centre shortly after the baby. But if the mother is the only person with parental responsibility, the consent process may have to take place by telephone, which is never satisfactory.

The risk profile of the baby is another important factor. If a baby is assessed as having a high chance of dying shortly after birth, it is particularly important for the mother to be in the same place as the baby throughout the child's short life. There are few things more awful than moving a sick baby to a facility distant from the mother, only for the child to die before the mother can be transferred. Furthermore, participating in a decision about switching to palliative care is the hardest situation a parent can be in, so parents need to hear expert views if they are to be sure that palliation, rather than a heroic but futile procedure, is the right option.

If the regional centre is not geographically very distant from the parental home, re-booking the mother for delivery there is relatively straightforward and overcomes the difficulty of not having the mother on site for early postnatal procedures on her child. However, if the distance between the local maternity facility and the regional centre is great, the issues are very different. One option is to book the mother for induction of labour at, or close to, the specialist centre at 38 weeks, in the hope that she does not go into labour sooner than this. Another is a planned caesarean section at around 38 weeks, but in this scenario the parents need to know about the increased potential for neonatal respiratory illness to complicate the baby's course and perhaps delay surgery. Admitting the mother to the antenatal ward in the specialist centre a week or 2 before the estimated date of delivery to await natural labour is a possibility - but this is extraordinarily disruptive to a family if there are other children to be looked after, and can cost the father his job.

As if these choices were not complicated enough, we also have to be realistic about the nature of prenatal diagnosis. Even with the most expert fetal medicine specialists scanning the fetus with ultrasound, and the increasing use of MRI, the unexpected still happens: babies with apparently fearsome lesions turn out to be relatively well, and babies with apparently minor issues can turn out to have lethal malformations. This uncertainty is not always effectively transmitted to parents, and we all need to get better at it.

So to participate effectively in the decision making, and to achieve delivery in the place that after all deliberations is the 'best' one for them, families need to know about the uncertainties, understand about consent issues, have a view about mode of delivery and have some knowledge of the non-clinical facilities at the specialist centre (such as hostel accommodation or overnight parent rooms). Every family in this situation needs a plan A and a plan B: $A$ is the plan for the best case scenario that is agreed by all parties, and B is the contingency plan to deal with the situation where the onset of labour happens earlier, or in some other place, than anticipated in plan A. Creating workable plans for each family can be difficult when there are so many clinical stakeholders involved, but with imagination and a little forethought we should be able to get it right most of the time.

Competing interests None.

Provenance and peer review Not commissioned; externally peer reviewed.

Open Access This is an Open Access article distributed in accordance with the Creative Commons Attribution Non Commercial (CC BY-NC 3.0) license, which permits others to distribute, remix, adapt, build upon this work non-commercially, and license their derivative works on different terms, provided the original work is properly cited and the use is noncommercial. See: http://creativecommons.org/licenses/ by-nc/3.0/ 


\section{Editorial}

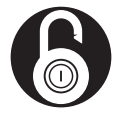

OPEN ACCESS

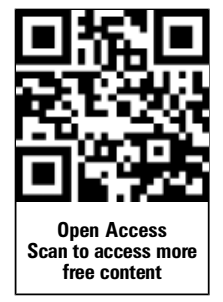

Received 06 February 2012

Accepted 20 February 2012

Published Online First 13 December 2012

\section{SLinked}

- http://dx.doi.org/10.1136/fetalneonatal-2011-300488
Arch Dis Child Fetal Neonatal Ed 2013:98:F190-F191. doi:10.1136/fetalneonatal-2012-301837

\section{REFERENCE}

1 Kelsall W, Anagnostou K, Messenger L, et al. Outcome of infants with prenatally diagnosed congenital heart disease delivered outside specialist paediatric cardiac centres. Arch Dis Child Fetal Neonatal Ed 2013;98:F218-F221.

To cite Platt MPW. Arch Dis Child Fetal Neonatal Ed 2013;98:F190-F191. 